\title{
Low Temperature Properties of the Hierarchical Classical Vector Model
}

\author{
Ricardo Schor and Michael O'Carroll \\ Departamento de Física do ICEx, Universidade Federal de Minas Gerais, Belo Horizonte, \\ Minas Gerais, Brasil
}

Received April 20, 1990; in revised form December 18, 1990

\begin{abstract}
We obtain low temperature properties of the classical vector model in a hierarchical formulation in three or more dimensions. We consider the lattice model in a zero or non-zero magnetic field, where the single site spin variable $\phi \in R^{v}$ has a density proportional to $e^{-\lambda\left(\phi^{2}-1\right)^{2}}$ for large $\lambda \leqq \infty$. Using renormalization group methods we obtain a convergent expansion for the free energy with zero magnetic field. For non-zero fields a shift formula is used to obtain the effective action generated by the renormalization group transformation (RGT). To obtain the pure state zero field free energy and spontaneous magnetization we take the thermodynamic limit together with the zero field limit at a specified rate. The spontaneous magnetization, $m$, is calculated, is non-zero and the pure state free energy coincides, as expected, with the zero field free energy. Also the sequence of zero field actions does not have a limit but we show that the sequence of actions generated from the original action shifted by $m$ does; the limiting action corresponds to a non-canonical Gaussian fixed point of the RGT.
\end{abstract}

\section{Introduction and Results}

Consider the $d$-dimensional lattice classical vector model with partition function given by

$$
Z=\int e^{\beta\left[1 / 2(\phi, \Delta \phi)+\left(h, \phi_{1}\right)\right]} \pi \delta\left(|\phi(x)|^{2}-1\right) d \phi(x),
$$

where $\phi(x)=\left(\phi_{1}(x), \ldots, \phi_{v}(x)\right) \in R^{v}$ and $\Delta$ is the lattice Laplacian. We want to obtain low temperature (large $\beta$ ) properties of a hierarchical formulation of this model. Formal high and low expansions have been obtained for physical quantities such as the free energy, magnetization and correlation functions for this model $[1,2]$. Rigorous low temperature results have been obtained in $[3,4]$ for $d=2$ and in [5] for $d=3, v=2$. For $d=3, v=2$ there is spontaneous magnetization (see [6]) and the truncated correlation functions for $h=0$ are expected, according to the Goldstone picture, to exhibit canonical $|x-y|^{-(d-2)}$ falloff perpendicular to 
the field; $|x-y|^{-2(d-2)}$ falloff parallel to the field. This model can be analyzed using renormalization group (RG) methods as in [7], where the large field problem solved in [8] is present. Here we analyze the model in a hierarchical formulation using RG methods in the spirit of $[9,10]$. See also [11-13] for results on other hierarchical models. Specifically in the full model the change of variables $\phi \rightarrow \beta^{1 / 2} \phi$ is made, the fixed spin condition is relaxed and the Laplacian is replaced by a hierarchical one, i.e. the partition function on the lattice $\Lambda_{N}=\left[-\frac{L^{N}}{2}, \frac{L^{N}}{2}\right]^{d}$ is given by

$$
Z_{N}(h, \beta)=\int \exp \left(\beta^{1 / 2} h \sum_{x \in \Lambda_{n}} \phi_{1}(x)-\frac{\lambda}{\beta} \sum_{x \in \Lambda_{n}}\left(\phi(x)^{2}-\beta\right)^{2}\right) d \mu_{N}(\phi) .
$$

$d \mu_{N}(\phi)$ is a Gaussian probability measure with covariance given by the inverse of the hierarchical Laplacian (see below). We use the RG of [10] which allows us to write

$$
\begin{aligned}
Z_{N} & =\int \prod_{x \in \Lambda_{N}} e^{-V(\phi(x))} d \mu_{N}(\phi)=\ldots \int \prod_{x \in \Lambda_{1}} e^{-R^{N-1} V(\phi(x))} d \mu_{1}(\phi) \\
& =\int e^{-R^{N} V(\phi)} d \mu_{0}(\phi),
\end{aligned}
$$

where $\Lambda_{m}=\left[-\frac{L^{m}}{2}, \frac{L^{m}}{2}\right]^{d}, L$ odd; $d \mu_{m}$ is a Gaussian probability measure with covariance $G_{m}$. The final integral is over a single site and $G_{0}=\left(1-L^{-(d-2)}\right)^{-1} \cdot G_{m}$ is given by

$$
G_{m}(x, y)=\left(1-L^{2-d}\right)^{-1} L^{(2-d)(N(x, y)-1)},
$$

for all $x, y \in \Lambda_{m}$ and $N(x, y)=\min \left\{n=\{1,2,3, \ldots\} ;\left[L^{-n} x\right]=\left[L^{-n} y\right]\right\}$, where for any $u \in R^{d},[u]$ is the element of $Z^{d}$ such that $\left(-\frac{1}{2}\right) \leqq u_{i}-[u]_{i}<\frac{1}{2}$. The $G_{n}$ satisfy the recursion relation

$$
G_{n}(L x+u, L y+v)=L^{(2-d)} G_{n-1}(x, y)+\delta_{n-1}(x, y),
$$

for all $x, y \in \Lambda_{n-1}$ and $u, v$ such that $-\frac{L}{2} \leqq u_{\alpha}, v_{\alpha}<\frac{L}{2} ; \delta_{n-1}(x, y)$ is the Kronecker $\delta$.

The relation (1.3) is derived using the decompositions

$$
\begin{gathered}
\phi(L x+u)=L^{-1 / 2(d-2)} \phi^{\prime}(x)+\eta(x), \quad x \in \Lambda_{m}, \\
d \mu_{m+1}(\phi)=d \mu_{m}\left(\phi^{\prime}\right) d \varrho_{m}(\eta),
\end{gathered}
$$

where $d \varrho_{m}(\eta)=\prod_{x \in A_{m}} d \mu(\eta(x)$ and $d \mu(\eta(x))$ is a Gaussian probability measure with covariance 1. In Eq. (1.3) the renormalization group transformation (RGT) $R$ is defined by

$$
e^{-R V(\phi)}=\int e^{-L^{1 d} V\left(L^{-} \overline{2}^{(d-2)} \phi+\eta\right)} d \mu(\eta) .
$$

We give some important properties of the RGT $R$ easily obtained by induction: 1. Commutation with translations. Define the translation by $\phi_{0} \in R^{v}$ by $T_{\phi_{0}} w(\phi)$ $=w\left(\phi-\phi_{0}\right)$. We find that

$$
R^{n} T_{L}-\frac{n}{2}(d-2)_{\phi_{0}}=T_{\phi_{0}} R^{n}
$$


2. Linear shift formula. Let $I(\phi)=\phi_{1}$ then

$$
\begin{gathered}
R^{n}\left(V-\beta^{1 / 2} h I\right)=T_{-L} d_{L} \frac{n}{2}(d+2)\left(\frac{1-L^{-2 n}}{L^{2}-1}\right) \beta^{1 / 2} h^{R^{n} V} \\
-L^{n / 2(d+2)} \beta^{1 / 2} h I-\frac{1}{2} L^{(n+1) d}\left(\frac{L^{2 n}-1}{L^{2}-1}\right) \beta h^{2}
\end{gathered}
$$

The RGT $R$ has Gaussian fixed points given by $V(\phi)=\sum_{i=1}^{v} c_{i} \phi_{i}^{2}$, where $c_{i}=0$ or $\frac{1}{2} \frac{L^{2}-1}{L^{d}}$. At the fixed point the two-point correlation functions behave as

$$
\left\langle\phi_{i}(x) \phi_{i}(y)\right\rangle \underset{|x-y| \rightarrow \infty}{\longrightarrow} \begin{cases}|x-y|^{-(d-2)}, & c_{i}=0 \\ |x-y|^{-(d+2)}, & c_{i} \neq 0 .\end{cases}
$$

We call the fixed point associated with $c_{i}=0\left(c_{i} \neq 0\right)$ a canonical (non-canonical) fixed point.

In this paper we will be concerned with the zero magnetic field free energy $F=\lim _{N \rightarrow \infty} L^{-N d} \ln Z_{N}$ and with the spontaneous magnetization in a pure state at zero magnetic field.

There are several approaches for constructing the $h=0$ pure state, i.e. by imposing boundary conditions or by first taking the $h \neq 0$ thermodynamic limit followed by the $h \rightarrow 0$ limit. In the hierarchical model it is technically simpler to take the thermodynamic limit together with the $h \rightarrow 0$ limit. To be more precise define the finite volume free energy by

$$
F_{N}(h)=-\beta^{-1} L^{-N d} \ln Z_{n}(h)
$$

and the finite volume magnetization per site by

$$
m_{N}(h) \equiv-\frac{\partial F_{N}(h)}{\partial h}
$$

We choose the sequence $\left\{h_{N}\right\}$ of magnetic fields so that $h \simeq L^{-2 N}$ (see Theorem 3 for the precise behavior). We will state in Theorem 3 below, after additional notation is introduced, the existence of the limits

$$
F_{+} \equiv \lim _{N \rightarrow \infty} F_{N}\left(h_{N}\right)
$$

and

$$
m \equiv \lim _{N \rightarrow \infty} m_{N}\left(h_{N}\right) .
$$

Furthermore $F_{+}=F$ and $m \neq 0 . F_{+}(m)$ is the pure state $h=0$ free energy (spontaneous magnetization). In a subsequent paper we will apply this procedure to generate correlation functions and verify that the construction indeed yields a pure state.

Using the linear shift formula (1.9) properties of $F_{N}(h)$ are obtained from the zero field $Z_{N}$ and the study of $Z_{N}$ is reduced to a control of the sequence $R^{n} V$ which depend on $\phi$ only through $|\phi|$. The starting $V$ is $\frac{\lambda}{\beta}\left(\phi^{2}-\beta\right)^{2}$. The sequence $R^{n} V$ is analyzed using perturbation theory in the small field region $\left(|\phi|-\beta_{n}^{1 / 2} \mid<\beta_{n}^{\alpha}, 0<\alpha\right.$ 
small), and a stability bound in the large field region. In the small field region we write $V$ as a power series in $|\phi|-\beta^{1 / 2}$ with leading term $4 \lambda\left(|\phi|-\beta^{1 / 2}\right)^{2}$. A flow of $\beta$ and $\lambda$ is obtained. Letting $\beta=\beta_{0}, \lambda=\lambda_{0}$ we find

$$
\beta_{n+1} \simeq L^{(d-2)} \beta_{n}, \quad \lambda_{n+1} \approx \frac{L^{2} \lambda_{n}}{1+8 L^{d} \lambda_{n}}, \quad n \geqq 1
$$

and

$$
\lambda_{n} \underset{n \rightarrow \infty}{\longrightarrow} \frac{L^{2}-1}{8 L^{d}} \equiv \lambda^{*} .
$$

The limiting $\lambda=\infty$ can be allowed after the first step and $\lambda_{1}$ is already close to $\lambda^{*}=f\left(\lambda^{*}\right)$, the fixed point of the map $\lambda \mapsto f(\lambda)=L^{2} \lambda /\left(1+8 L^{d} \lambda\right)$.

We now state the main theorem on the properties of $R^{n} V$ for $h=0$. It is more convenient to state the results in terms of a normalized version of $R^{n} V$, which we denote by $V^{(n)}$, defined such that it vanishes at its minimum in the region of small fields. The constant $d_{n}=R^{n} V-V^{(n)}$ will be specified as part of the basic result given by

Theorem 1. Let $\beta, \lambda$, and $L$ be sufficiently large and let $\alpha$ be a small positive number, $0<\alpha<1 /[6(d-2)]$. There are sequences $\left\{\beta_{n}\right\},\left\{\lambda_{n}\right\}$ with $\beta_{0}=\beta$ and $\lambda_{0}=\lambda$ such that

a) $\lim \lambda_{n} \equiv \lambda=\frac{L^{2}-1}{8 L^{d}}$ and $\lim _{n \rightarrow \infty} L^{-n(d-2)} \beta_{n}=\gamma^{2}$.

b) The function $R^{n} V(\phi)$ has a minimum at $\phi=\beta_{n}^{1 / 2} \phi /|\phi|=\beta_{n}^{1 / 2} \hat{\phi}$.

c) Letting $\phi=\left(\sigma+\beta_{n}^{1 / 2}\right) \hat{\phi}, R^{n} V$ as a function of $\sigma$ is analytic in $\left\{\sigma \in C:|\sigma|<\beta_{n}^{\sigma}\right\}$ and $\sigma=0$ is the only minimum of $R^{n} V$ in that region.

d) Let $V^{(n)}=R^{n} V(\phi)-d_{n}$, where $d_{n}=R^{n} V\left(\beta_{n}^{1 / 2} \phi\right)$, then for $|\sigma|<\beta_{n}^{\alpha} V^{(n)}(\phi)=4 \lambda_{n} \sigma^{2}$ $+w_{n}(\sigma), \frac{d^{p}}{d \sigma^{p}} w_{n}(\sigma=0)=0$ or $0 \leqq p \leqq 2$, and $\left|w_{n}(\sigma)\right| \leqq k \beta_{n}^{3 \alpha-1 / 2}$ for a suitable ( $L$ dependent) constant $k$.

e) $\varrho_{n}(\phi) \equiv e^{-V^{(n)}(\phi)}$ is an entire function and

$$
\left|\varrho_{n}(\phi)\right| \leqq \exp \left[-\lambda^{*}\left(|\operatorname{Re} \phi|-\beta_{n}^{1 / 2}\right)^{2}+\frac{1}{2} L^{-(d-2)}|\operatorname{Im} \phi|^{2}\right] \text {. }
$$

f) $d_{n}=L^{n d} \sum_{j=0}^{n-1}\left(L^{-(j+1) d}\left(\log \left(1+8 L^{d} \lambda_{j}\right)^{1 / 2}+O\left(\beta_{j}^{-3 \alpha-1 / 2}\right)\right)\right.$, the $O(\cdot)$ term is independent of $j$ and $\lambda$.

To obtain an expansion for the $h=0$ free energy we use the relation

$$
Z_{N}=\int e^{-R^{N} V} d \mu_{0}=e^{-d_{N}} \int e^{-V^{(N)}} d \mu_{0}
$$

which gives

$$
F_{N}=\frac{1}{\beta} \frac{d_{N}}{L^{N d}}-\frac{1}{L^{N d}} \log \int e^{-V^{(N)}} d \mu_{0} .
$$

Using Theorem 1 to control $d_{N}$ and the above integral we have

Theorem 2.

$$
F=\frac{1}{\beta} \sum_{j=0}^{\infty} L^{-(j+1) d}\left(\log \left(1+8 \lambda_{j} L^{d}\right)^{1 / 2}+O\left(\beta_{j}^{3 \alpha-1 / 2}\right)\right) .
$$

Remark. This result displays the multi-scale nature of the expansion. 
The global upper bound in Theorem 1e) can be improved to establish the existence of the free energy for $h \neq 0$; stability bounds can be obtained by elementary methods, i.e. maximizing the integrand for the upper bound and using Jensen's inequality for the lower bound. Since we are interested only in the $h=0$ pure state, we will not pursue the $h \neq 0$ model further.

Now we include the magnetic field $h$ so that

$$
Z_{N}(h)=\int e^{-R^{N}\left(V-\beta h^{1 / 2} I\right)(\phi)} d \mu_{0} .
$$

Using the shift formula (1.9) and letting $h$ depend on the volume we obtain results for the pure state $h=0$ free energy and spontaneous magnetization given by

Theorem 3. Let

$$
h_{N}=\beta^{-1 / 2}\left[\left(\frac{L^{d}-1}{L^{2}-1}\right)-\left(\frac{L^{d}-L^{2}}{L^{2}-1}\right) L^{-2 N}\right]^{-1}\left(1-L^{2-d}\right) \cdot L^{-1 / 2 N(d+2)} \beta_{N}^{1 / 2} .
$$

Then

$$
\begin{gathered}
F_{+} \equiv \lim _{N \rightarrow \infty} F_{N}\left(h_{N}\right)=F \\
m \equiv \lim _{N \rightarrow \infty} \frac{\partial F_{N}}{\partial h}\left(h_{N}\right)=\beta^{-1 / 2} \lim _{n \rightarrow \infty} L^{-n / 2(d-2)} \beta_{n}^{1 / 2} \neq 0 .
\end{gathered}
$$

In Theorem 3 we have chosen the sequence $\left\{h_{N}\right\}$ so as to make the proof effortless; a range of $h_{N}$ 's can be permitted using more complicated estimates in the proof.

Although $R^{n} V$ does not have a limit we find that if the initial $V$ is translated by $\beta^{1 / 2} m$ then the sequence obtained by iterating with the RGT suitably renormalized converges to a non-canonical Gaussian fixed point.

We have, denoting the unit vector in the 1-direction by $e$.

Theorem 4. Let $V_{-\beta^{1 / 2 m e}}(\phi)=V\left(\phi+\beta^{1 / 2} m e\right)$ and let $V_{-\beta^{1 / 2} m e}^{(n)}=R^{n} V_{-\beta^{1 / 2} m e}-d_{n}$ with $d_{n}$ given by Theorem $1 f$. Then

$$
\lim _{n \rightarrow \infty} V_{-\beta^{1 / 2} m e}^{(n)}(\phi)=4 \lambda^{*} \phi_{1}^{2}
$$

uniformly on compact sets of $R^{v}$.

The above result indicates that the truncated correlation functions parallel to the spontaneous magnetization have long-range behavior controlled by the noncanonical Gaussian fixed point and the ones perpendicular decay canonically. Since the non-canonical fixed point is a property specific to the hierarchical model we do not expect the same falloff of the parallel correlation functions in the complete model.

We now describe the content and organization of the remainder of this paper. We prove Theorem 1 by induction. In Sect. II we give the first step proof; in Sect. III the proof for a general induction step is given and the proof of Theorem 1 is completed. Theorem 2 is proved in Sect. IV; Theorems 3 and 4 are proved in Sect. V. In Sect. VI we make some concluding remarks.

\section{Proof of Theorem 1 - First Step}

In this section we give the proof of Theorem 1 for $n=1$ which requires special treatment since $\lambda$ can be arbitrarily large. To analyze $V^{(1)}(\phi)$ we define $\sigma^{\prime} \in R$ by 
$\phi=\left(\sigma^{\prime}+L^{1 / 2(d-2)} \beta^{1 / 2}\right) \hat{\phi}$ and set $\xi=u \hat{\phi}+t, u \in R$, with $\hat{\phi} \cdot t=0$. In $e^{-R V^{(0)}}$ we pass to the minimum of the quadratic form in $u$ to obtain the representation

$$
\begin{aligned}
e^{-V^{(1)}}= & c_{0} \exp \left(-4 \lambda^{\prime} \sigma^{\prime 2}\right) \int \exp \left\{-\frac{1}{2}\left(u^{2}+t^{2}\right)\right. \\
& \left.-\frac{\lambda L^{d}}{\beta}\left[\left(u+\beta^{1 / 2}\right)^{2}+t^{2}-\beta\right]^{2}-\varrho\left(\frac{L^{-1 / 2(d-2)}}{1+8 \lambda L^{d}} \sigma^{\prime}, u, t^{2}\right)\right\} d u d t \\
\equiv & c_{0} \exp \left(-4 \lambda^{\prime} \sigma^{\prime 2}\right) I\left(\sigma^{\prime}\right),
\end{aligned}
$$

where

$$
c_{0}=e^{d_{1}}, \quad \lambda^{\prime}=\frac{L^{2} \lambda}{1+8 \lambda L^{d}}
$$

and, letting $\mathscr{L}=L^{-1 / 2(d-2)} /\left(1+8 \lambda L^{d}\right)$,

$$
\begin{aligned}
\varrho= & \frac{4 \lambda L^{d}}{\beta^{1 / 2}}\left(3 u^{2}+t^{2}+\frac{u^{3}}{\beta^{1 / 2}}+\frac{u^{2}}{\beta^{1 / 2}}\right) \mathscr{L} \sigma^{\prime} \\
& +\frac{4 \lambda L^{d}}{\beta^{1 / 2}}\left(3 u+\frac{3 u^{2}}{2 \beta^{1 / 2}}+\frac{t^{2}}{2 \beta^{1 / 2}}\right)\left(\mathscr{L} \sigma^{\prime}\right)^{2} \\
& +\frac{4 \lambda L^{d}}{\beta^{1 / 2}}\left(1+\frac{u}{\beta^{1 / 2}}\right)\left(\mathscr{L} \sigma^{\prime}\right)^{3}+\frac{\lambda L^{d}}{\beta}\left(\mathscr{L} \sigma^{\prime}\right)^{4} .
\end{aligned}
$$

We adopt the following notational convention: the integral in $t$ is restricted to $\hat{\phi} \cdot t=0$ and the integral includes the factor $(2 \pi)^{-v / 2}$. We analyze $I\left(\sigma^{\prime}\right)$ by splitting the integration into large and small field contributions. Letting $X^{c}=1-X$, where

$$
\begin{gathered}
\chi(u, t)= \begin{cases}1, & \text { if }|u|,|t|<\beta^{\alpha} \\
0, & \text { otherwise }\end{cases} \\
I\left(\sigma^{\prime}\right)=\int\left(\chi+\chi^{c}\right) \exp \{\ldots\} d u d t \equiv I_{0}\left(\sigma^{\prime}\right)+I_{c}\left(\sigma^{\prime}\right) .
\end{gathered}
$$

We have

Lemma II.1. If $\left|\sigma^{\prime}\right|<\left(2 L^{d-2} \beta\right)^{\alpha}$, then

$$
I_{c}\left(\sigma^{\prime}\right) \leqq c \frac{\beta^{1 / 2(v-1)}}{\sqrt{\lambda}} \exp \left(-\frac{\beta^{2 \alpha}}{4}\right),
$$

where $C$ is a constant.

Remark. Here and in the sequel different constants will usually be denoted by the same letter.

Proof of Lemma II.1. For $\left|\sigma^{\prime}\right|<\left(2 L^{d-2} \beta\right)^{\alpha}$ and $\alpha=1 / 6$ then

$$
\begin{aligned}
|\varrho| \leqq & \frac{\lambda L^{d}}{\beta} \frac{4 L^{-1 / 2(d-2)}}{1+8 \lambda L^{d}}\left|\sigma^{\prime}\right||u|\left(u^{2}+t^{2}\right)+\left(3 u^{2}+t^{2}\right) O\left(\beta^{\alpha-1 / 2}\right) \\
& +\left(3|u|+\frac{1}{2 \beta^{1 / 2}}\left(3 u^{2}+t^{2}\right)\right) O\left(\beta^{2 \alpha-1 / 2}\right)+\left(1+|u| / \beta^{1 / 2}\right)+O\left(\beta^{4 \alpha-1}\right) \\
\leqq & \frac{\lambda L^{d}}{2 \beta}\left(\left(u+\beta^{1 / 2}\right)^{2}+t^{2}-\beta\right)^{2}+\left(u^{2}+t^{2}\right)\left(\beta^{2 \alpha-1 / 2}\right)+O\left(\beta^{3 \alpha-1 / 2}\right)
\end{aligned}
$$


Thus

$$
\begin{aligned}
\left|I_{c}\left(\sigma^{\prime}\right)\right| \leqq & \exp \left[O\left(\beta^{3 \alpha-1 / 2}\right)\right] \int \chi^{c} \exp \left\{-\frac{1}{2}\left(1+O\left(\beta^{2 \alpha-1 / 2}\right)\right)\left(u^{2}+t^{2}\right)\right. \\
& \left.-\frac{\lambda L^{d}}{2 \beta}\left[\left(u+\beta^{1 / 2}\right)^{2}+t^{2}-\beta\right]^{2}\right\} d u d t,
\end{aligned}
$$

and for $\beta$ sufficiently large

$$
\left|I_{c}\left(\sigma^{\prime}\right)\right| \leqq \exp \left[O\left(\beta^{3 \alpha-1 / 2}\right)-\frac{1}{4} \beta^{2 \alpha}\right] J,
$$

where

$$
J \equiv \int \exp \left[-\frac{\lambda L^{d}}{2 \beta}\left(\xi^{2}-\beta\right)^{2}\right] d \xi \quad \text { and } \quad J \leqq c \beta^{1 / 2(N-1)} / \sqrt{\lambda} \text { for } \frac{\lambda \beta L^{d}}{2}>1 .
$$

Concerning $I_{0}\left(\sigma^{\prime}\right)$ we have

Lemma II.2. For $\left|\sigma^{\prime}\right|<\left(2 L^{d-2} \beta\right)^{\alpha}$ and $\beta$ large

$$
\log I_{0}\left(\sigma^{\prime}\right)=\log I_{0}(O)-g_{1}\left(\sigma^{\prime}\right)
$$

where

$$
I_{0}(O)=\left(1+8 \lambda L^{d}\right)^{-1 / 2}\left(1+O\left(\beta^{3 \alpha-1 / 2}\right) \geqq C\left(1+8 \lambda L^{d}\right)^{-1 / 2},\right.
$$

$g_{1}\left(\sigma^{\prime}\right)$ is analytic and $\left|g_{1}\left(\sigma^{\prime}\right)\right|<\subset \beta^{3 \alpha-1 / 2}$.

Proof of Lemma II.2. From Eq. (2.1) if $|u|,|t|<\beta^{\alpha}$ then $|\varrho| \leqq O\left(\beta^{3 \alpha-1 / 2}\right)$. Write $I_{0}\left(\sigma^{\prime}\right)$ $=\int \chi e^{-v-\varrho} d u d t$, where $v=\frac{1}{2}\left(u^{2}+t^{2}\right)+\frac{\lambda L^{d}}{\beta}\left[\left(u+\beta^{1 / 2}\right)^{2}+t^{2}-\beta\right]^{2}$ and $I_{0}\left(\sigma^{\prime}\right)$ $=I_{0}(0) \int e^{-\varrho} d \mu$, where $d \mu=\chi e^{-v} d u d t / \int \chi e^{-v} d u d t$. Thus $\log I_{0}\left(\sigma^{\prime}\right)=\log I_{0}(O)$ $-g_{1}\left(\sigma^{\prime}\right)$, where $g_{1}\left(\sigma^{\prime}\right) \equiv \log \int e^{-\varrho} d \mu$. As $\left|\int\left(e^{-e}-1\right) d \mu\right| \leqq c \quad O\left(\beta^{3 \alpha-1 / 2}\right), \quad\left|g_{1}\left(\sigma^{\prime}\right)\right|$ $\leqq O\left(\beta^{3 \alpha-1 / 2}\right)$ and is analytic on $\left|\sigma^{\prime}\right|<\left(2 \mathrm{~L}^{d-2} \beta\right)^{\alpha}$. To analyze $I_{0}(O)$ we make the change of variables $(u, t)(s, q)$ where $q=t$ and $s=\frac{1}{2 \beta^{1 / 2}}\left[\left(u+\beta^{1 / 2}\right)^{2}+t^{2}-\beta\right]$ for $u, t<\beta^{\alpha}$,

$$
I_{0}(O)=\frac{1}{\left(1+8 \lambda L^{d}\right)^{1 / 2}}-\int \tilde{\chi}^{c} \exp \left[-\frac{1}{2}\left(1+8 \lambda L^{d}\right) s^{2}-\frac{1}{2} q^{2}\right] d s d q+\frac{\left(\beta^{3 \alpha-1 / 2}\right)}{\left(1+8 \lambda L^{d}\right)^{1 / 2}},
$$

where $\tilde{\chi}^{c}=1-\tilde{\chi} \cdot \tilde{\chi}$ is the characteristic function of the region in $(s, q)$ space corresponding to the region $|u|, t<\beta^{\alpha}$ in $(u, t)$ space. Estimating the integral by $c e^{-\beta^{2 \alpha / 16}}\left(1+8 \lambda L^{d}\right)^{1 / 2}$, we arrive at

$$
I_{0}(O)=\left(1+8 \lambda L^{d}\right)^{-1 / 2}\left(1+O\left(1+O\left(\beta^{3 \alpha-1 / 2}\right)\right) \geqq c\left(1+8 \lambda L^{d}\right)^{-1 / 2} .\right.
$$

for large $\beta$ with $c$ strictly positive.

Combining Lemmas II.1 and II.2 we have

Lemma II.3. For $\left|\sigma^{\prime}\right|<\left(2 L^{d-2} \beta\right)^{\alpha}$

$$
I\left(\sigma^{\prime}\right)=I_{0}(O) e^{-g_{2}\left(\sigma^{\prime}\right)},
$$

where $g_{2}\left(\sigma^{\prime}\right)$ is analytic and $\left|g_{2}\left(\sigma^{\prime}\right)\right|<O\left(\beta^{3 \alpha-1 / 2}\right)$. 
Towards obtaining the final small field representation $V^{(1)}(\phi)$ we have

Lemma II.4. For $\left|\sigma^{\prime}\right|<\left(2 L^{d-2} \beta\right)^{\alpha}, V^{(1)}(\phi)=c+4 \lambda^{\prime} \sigma^{\prime 2}+g_{2}\left(\sigma^{\prime}\right)$, where $g_{2}\left(\sigma^{\prime}\right)$ is analytic and $\left|g_{2}\left(\sigma^{\prime}\right)\right|<C \beta^{3 \alpha-1 / 2}$. Furthermore $V^{(1)}$ has a unique minimum, $\sigma_{0}$, for $\left|\sigma^{\prime}\right|$ $<\left(\frac{3}{2} L^{d-2} \beta\right)^{\alpha}$ and $\left|\sigma_{0}\right|=O\left(\beta^{2 \alpha-1 / 2}\right)$.

Proof of Lemma II.4. From Eq. (2.1) and Lemmas 2.2 and 2.3 we have for $\left|\sigma^{\prime}\right|$ $<\left(2 L^{d-2} \beta\right)^{\alpha}, V^{(1)}(\phi)=C+4 \lambda^{\prime} \sigma^{\prime 2}+g_{2}\left(\sigma^{\prime}\right)$. Using Cauchy estimates for $g_{2}^{\prime}\left(\sigma^{\prime}\right)$ and Rouche's Theorem for $d V^{(1)} / d \sigma^{\prime}$ gives the result.

Finally Taylor expanding $V^{(1)}(\phi)$ around $\sigma_{0}$, letting $\sigma=\sigma^{\prime}-\sigma_{0}$ and defining $V^{(1)}(\phi)$ to be zero at its minimum $\sigma=0$ we have

\section{Lemma II.5.}

$$
\begin{gathered}
V^{(1)}(\phi)=4 \lambda_{1} \sigma^{2}+w_{1}(\sigma),|\sigma|<\left(\frac{5}{4} L^{(d-2)} \beta\right)^{2}, \quad \text { where } \phi=\left(\sigma+\beta_{1}^{1 / 2}\right) \hat{\phi} \\
\frac{1}{2} \lambda^{*} \leqq \lambda_{1}=\lambda^{\prime}+O\left(\beta^{\alpha-1 / 2}\right) \leqq 3 \lambda^{*}, \beta_{1}^{1 / 2}=L^{1 / 2(d-2)} \beta^{1 / 2}+\sigma_{0} .
\end{gathered}
$$

$w_{1}(\sigma)$ is analytic in $|\sigma|<\beta_{1}^{\alpha}, \frac{d^{n} w_{1}}{d \sigma^{n}}(0)=0$ for $n=0,1,2$ and $\left|w_{1}(\sigma)\right|<k \beta_{1}^{3 \alpha-1 / 2}, k$ an $L$ dependent constant.

Proof of Lemma II.5. For large $\beta$ and $\left|\sigma^{\prime}-\sigma_{0}\right|<\left|\frac{5}{4} L^{d-2} \beta\right|^{\alpha}$ we have the Taylor series

$$
V^{(1)}(\phi)=C+4 \lambda_{1}\left(\sigma^{\prime}-\sigma_{0}\right)^{2}+w_{1}\left(\sigma^{\prime}-\sigma_{0}\right),
$$

where using Cauchy estimates

$$
\lambda_{1}=\lambda^{\prime}+O\left(\beta^{\alpha-1 / 2}\right),\left|w_{1}\left(\sigma^{\prime}-\sigma_{0}\right)\right| \leqq \beta^{3 \alpha-1 / 2} .
$$

Writing $\sigma=\sigma^{\prime}-\sigma_{0}$ and defining $V^{(1)}(\phi)$ to be zero at its minimum $\sigma=0$, we have for $|\sigma|<\left(\frac{5}{4} L^{d-2} \beta\right)^{\alpha}, \quad V^{(1)}(\phi)=4 \lambda_{1} \sigma^{2}+w_{1}(\sigma)$ and $\phi=\left(\sigma^{\prime}+L^{1 / 2(d-2)} \beta^{1 / 2}\right) \phi=\left(\sigma+\sigma_{0}\right.$ $\left.+L^{1 / 2(d-2)} \beta^{1 / 2}\right) \phi \equiv\left(\sigma+\beta_{1}^{1 / 2}\right) \phi$, where $\beta_{1}^{1 / 2}=L^{1 / 2(d-2)} \beta^{1 / 2}+\sigma_{0}=L^{1 / 2(d-2)} \beta^{1 / 2}$ $+O\left(\beta^{2 \alpha-1 / 2}\right)<\left(\frac{5}{4} L^{(d-2)} \beta\right)^{1 / 2}$ for large $\beta$.

For the constant $d_{1}$ we have

Lemma II.6. $d_{1}=\frac{1}{2} \ln \left(1+8 \lambda L^{d}\right)+O\left(\beta^{3 \alpha-1 / 2}\right)$.

Proof of Lemma II.6. From $e^{-V^{(1)}(\phi)}=e^{-R V(\phi)} e^{R V\left(\beta_{1}^{1 / 2} \phi\right)}=e^{-R V(\phi)} e^{d_{1}}$ and Eq. (2.1) we have $e^{-d_{1}}=e^{-4 \lambda^{\prime} \sigma^{\prime 2}} I\left(\sigma^{\prime}\right)$, where $\sigma^{\prime}=\beta_{1}^{1 / 2}-L^{1 / 2(d-2)} \beta^{1 / 2}=O\left(\beta^{2 \alpha-1 / 2}\right)$. Using Lemmas II. 2 and II. 3 the result follows.

We now consider the global upper bound for real fields. In the large field region note that, using Lemma II.6,

$$
e^{-V^{(1)}(\phi)}=e^{d 1} e^{-R V(\phi)}=\frac{1}{\left(1+8 \lambda L^{d}\right)^{1 / 2}} e^{O\left(\beta^{3 \alpha-1 / 2}\right)} e^{-R V(\phi)} .
$$

The global large field upper bound follows from an upper bound on $e^{-\boldsymbol{R} V}$, where in the integrand of $e^{-R V}$ we use the inequality

We have

$$
V(\phi)=\frac{\lambda}{\beta}\left(\phi^{2}-\beta\right)^{2}>\lambda\left(|\phi|-\beta^{1 / 2}\right)^{2} .
$$

Lemma II.7. If ||$\phi\left|-\beta_{1}^{1 / 2}\right|>\frac{1}{2} \beta_{1}^{\alpha}$, then $e^{-R V(\phi)} \leqq e^{-\lambda\left(|\phi|-\beta_{1}^{1 / 2}\right)^{2}}$. 
Proof of Lemma II.7. Using the inequality in Eq. (2.2) and making a translation we have

$$
\begin{aligned}
e^{-\boldsymbol{R} V(\phi)} \leqq & \exp \left[-\frac{1}{2} L^{-(d-2)}|\phi|^{2}-L^{d} \lambda \beta\right] \int \exp \left[-\frac{1}{2}\left(1+2 L^{d} \lambda\right) \xi^{2}\right. \\
& \left.+|\xi|\left(L^{-1 / 2(d-2)}|\phi|+2 L^{d} \lambda \beta^{1 / 2}\right)\right] d \xi \\
\leqq & C\left(|\phi|+\beta_{1}^{1 / 2}+\left(\beta^{2 \alpha-1 / 2}\right)\right)^{\nu-1} \\
\times & \exp \left[-\frac{\lambda L^{2}}{1+2 \lambda L^{d}}\left(|\phi|-\beta_{1}^{1 / 2}+O\left(\beta^{2 \alpha-1 / 2}\right)\right)^{2}\right]
\end{aligned}
$$

For large $\beta$ and $\lambda \geqq \lambda^{*} / 2$.

As ||$\phi\left|-\beta_{1}^{1 / 2}\right| \geqq \frac{1}{2} \beta_{1}^{\alpha}$ we have

$$
\begin{aligned}
e^{-V^{(1)}(\phi)} \leqq & \exp \left[-\lambda^{*}\left(|\phi|-\beta_{1}^{1 / 2}\right)^{2}\right] \subset\left(|\phi|-\beta_{1}^{1 / 2}\right)^{(\nu-1)} \\
& \times \exp \left\{-\left[\frac{\lambda L^{2}}{1+2 \lambda L^{d}}\left(1+O\left(\beta^{\alpha-1 / 2}\right)\right)-\lambda^{*}\right]\left(|\phi|-\beta_{1}^{1 / 2}\right)^{2}\right\} .
\end{aligned}
$$

Now if $\lambda \geqq \frac{\lambda^{*}}{2}$ then recalling that $\lambda^{*}=\frac{L^{2}-1}{8 L^{d}}$ we have $\frac{\lambda L^{2}}{1+2 \lambda L^{d}} \geqq \frac{9}{4} \lambda^{*}$. Hence for $\beta$ large,

$$
\begin{aligned}
e^{-V^{(1)}(\phi)} \leqq & e^{-\lambda^{*}\left(|\phi|-\beta_{1}^{1 / 2}\right)^{2}} C|| \phi\left|-\beta_{1}^{1 / 2}\right|^{2 / \alpha(v-1)} \\
& \times \exp \left\{-\frac{11}{16} \lambda^{*}\left(|\phi|-\beta_{1}^{1 / 2}\right)^{2}\right\} \leqq e^{-\lambda^{*}\left(|\phi|-\beta_{1}^{1 / 2}\right)^{2}}
\end{aligned}
$$

To obtain the global upper bound in the small field region we use the representation and bounds of Lemma II.5 obtaining

Lemma II.8. If ||$\phi\left|-\beta_{1}^{1 / 2}\right|<\frac{1}{2} \beta_{1}^{\alpha}$, then

$$
e^{-V^{(1)}(\phi)} \leqq e^{-\lambda^{*}\left(|\phi|-\beta_{1}^{1 / 2}\right)^{2}} .
$$

Proof of Lemma II.8. For ||$\phi\left|-\beta_{1}^{1 / 2}\right|<\frac{1}{2} \beta_{1}^{\alpha}$,

$$
e^{-V^{(1)}(\phi)}=e^{-\lambda^{*}\left(|\phi|-\beta_{1}^{1 / 2}\right)^{2}} \exp \left[-\left(4 \lambda_{1}-\lambda^{*}\right) \sigma^{2}-w_{1}(\sigma)\right] .
$$

For large $\beta, \lambda_{1} \geqq \frac{9}{20} \lambda^{*}$. The function $w_{1}(\sigma) / \sigma^{2}$ is analytic on $|\sigma|<\beta_{1}^{\alpha}$ and thus, by the maximum principle, $\left|w_{1}(\sigma) / \sigma^{2}\right| \leqq k \beta_{1}^{\alpha-1 / 2}<\frac{4}{5} \lambda^{*}$. This implies $e^{-V^{(1)}(\phi)}$ $\leqq e^{-\lambda^{*}\left(|\phi|-\beta_{1}^{1 / 2}\right)^{2}}$.

To obtain the global upper bound for complex $\phi$ let $\varrho^{(1)}(\phi)$ be the extension of $e^{-V^{(1)}(\phi)}$ to complex $\phi$, i.e.

$$
\begin{aligned}
& \varrho^{(1)}(\phi)=e^{-R V(\phi)} e^{d_{1}} \\
& =e^{1 / 2 L^{-(d-2)}(\operatorname{Im} \phi)^{2}} e^{d_{1}} \int e^{-L^{d} V^{(0)}(\xi)-1 / 2\left(\xi-L^{-1 / 2(d-2)} \operatorname{Re} \phi\right)^{2}} \\
& \times e^{i\left(\xi-L^{-1 / 2(d-2)} \operatorname{Re} \phi\right) L^{-1 / 2(d-2)}(\operatorname{Im} \phi)} d \xi .
\end{aligned}
$$

Thus

$$
\varrho^{(1)}(\phi) \leqq e^{V^{(1)}(\operatorname{Re} \phi)+1 / 2 L^{-(d-2)}(\operatorname{Im} \phi)^{2}} \leqq e^{-\lambda^{*}\left(|\operatorname{Re} \phi|-\beta_{1}^{1 / 2}\right)^{2}+1 / 2 L^{-(d-2)}(\operatorname{Im} \phi)^{2}},
$$

and we have completed the proof of Theorem 1 for the first step. 


\section{Proof of Theorem I. General Induction Step}

In this section we prove a general induction step and complete the proof of Theorem I. Assume we are given a real analytic function $V(\phi)$ and two positive numbers $\beta, \lambda$ such that

a) $V(\phi)$ has a minimum at $\phi=\beta^{1 / 2} \hat{\phi}$,

b) Letting $\phi=\left(\sigma+\beta^{1 / 2}\right) \hat{\phi}, V$ as a function of $\sigma$ is analytic on $\left\{\sigma \in \mathbb{C}:|\sigma|<\beta^{\alpha}\right\}$ and $\sigma=0$ is the only minimum of $V$ in this region,

c) $V(\phi)=4 \lambda \sigma^{2}+w(\sigma)$ on $|\sigma|<\beta^{\alpha}: \frac{d^{p} w}{d \sigma^{p}}(\sigma=0)=0$ for $0 \leqq p \leqq 2$ and $|w(\sigma)|$ $\leqq k \beta^{3 \alpha-1 / 2}$,

d) $\varrho(\phi)=e^{-V(\phi)}$ extends to an entire function of $\phi$ and

$$
|\varrho(\phi)| \leqq \exp \left[-\lambda^{*}\left(|\operatorname{Re} \phi|-\beta^{1 / 2}\right)^{2}+\frac{1}{2} L^{-(d-2)}(\operatorname{Im} \phi)^{2}\right] .
$$

We will show that if $\beta$ is sufficiently large (depending only on $L$ ), if $\frac{\lambda^{*}}{2} \leqq \lambda \leqq 3 \lambda^{*}$ and if $V^{\prime}(\phi)$ is defined by

$$
e^{-V^{\prime}(\phi)}=c e^{-R V(\phi)}
$$

with a proper choice of normalization $c$, then $V^{\prime}(\phi)$ satisfies properties a)-d) with new constants $\beta^{\prime}, \lambda^{\prime}$ such that $\beta^{\prime 1 / 2}=L^{1 / 2(d-2)} \beta+O\left(\beta^{2 \alpha-1 / 2}\right)$ and $\lambda^{\prime}=\frac{\lambda L^{2}}{1+8 \lambda L^{d}}$ $+O\left(\beta^{\alpha-1 / 2}\right)$. If $\beta$ is large clearly $\beta^{1 / 2}>\frac{1}{2} L^{1 / 2(d-2)} \beta^{1 / 2}>\beta^{1 / 2}$ and $\frac{\lambda^{*}}{2} \leqq \lambda^{\prime} \leqq 3 \lambda^{*}$, therefore the process can be repeated starting with $V^{\prime}(\phi)$. Since $V^{(1)}(\phi)$ satisfies the hypotheses a)-d) we see that by choosing the initial $\beta$ large enough and $\lambda \geqq \lambda^{*} / 2$ we can construct the whole sequence $V^{(n)}(\phi)$, together with $\beta_{n}, \lambda_{n}$ such that

$$
\begin{gathered}
\left|\beta_{n+1}^{1 / 2}-L^{1 / 2(d-2)} \beta_{n}^{1 / 2}\right| \leqq C \beta_{n}^{2 \alpha-1 / 2}, \\
\left|\lambda_{n+1}-\frac{\lambda_{n} L^{2}}{1+8 \lambda_{n} L^{d}}\right| \leqq C \beta_{n}^{\alpha-1 / 2},
\end{gathered}
$$

with $C$ depending only on $L$.

We now begin the analysis of $V^{\prime}(\phi)$. Write $\phi=\left(\sigma^{\prime}+L^{1 / 2(d-2)} \beta^{1 / 2}\right) \hat{\phi}$ and $\xi=u \hat{\phi}+t, \hat{\phi} \cdot t=0$ so that $L^{-1 / 2(d-2)} \phi+\xi=\left(L^{-1 / 2(d-2)} \sigma^{\prime}+\beta^{1 / 2}+u\right) \hat{\phi}+t$. Also write $V\left(\left(L^{-1 / 2(d-2)} \sigma^{\prime}+u+\beta^{1 / 2}\right) \hat{\phi}+t\right) \equiv 4 \lambda\left(L^{-1 / 2(d-2)} \sigma^{\prime}+u\right)^{2}+\widetilde{V}\left(\left(L^{-1 / 2(d-2)} \sigma^{\prime}+u\right.\right.$ $\left.\left.+\beta^{1 / 2}\right) \hat{\phi}+t\right)$.

Substituting $V$ in the integral for $e^{-V^{\prime}}$ and, as in the first step, passing to the minimum in the quadratic form in $u$ we get, letting $\lambda_{L} \equiv \frac{L^{2} \lambda}{1+8 \lambda L^{d}}$,

$$
\begin{aligned}
e^{-V^{\prime}(\phi)}= & C e^{-4 \lambda_{L} \sigma^{\prime 2}} \int \exp \left\{-\frac{1}{2}\left(1+8 \lambda L^{d}\right) u^{2}-\frac{t^{2}}{2}\right. \\
& \left.-L^{d} \tilde{V}\left(\left(\frac{L^{-1 / 2(d-2)} \sigma^{\prime}}{1+8 \lambda L^{d}}+u+\beta^{1 / 2}\right) \hat{\phi}+t\right)\right\} d u d t \equiv C e^{-4 \lambda_{L} \sigma^{\prime 2}} I\left(\sigma^{\prime}\right) .
\end{aligned}
$$


We decompose the integral as, letting $\chi^{c}=1-\chi$,

We have

$$
\begin{aligned}
I\left(\sigma^{\prime}\right) & =\int\left(\chi+\chi^{c}\right) \exp \{\ldots\} d u d t \equiv I_{0}\left(\sigma^{\prime}\right)+I_{c}\left(\sigma^{\prime}\right), \\
\chi(u, t) & = \begin{cases}1, & \text { if }|u|,|t|<L^{-1 / 2(d-1 / 3)} \beta^{\alpha}, \\
0, & \text { otherwise }\end{cases}
\end{aligned}
$$

Lemma III.1. If $0<\alpha<\frac{1}{6(d-2)}$ and $\left|\sigma^{\prime}\right|<\left(2 L^{d-2} \beta\right)^{\alpha}$, then $I_{c}\left(\sigma^{\prime}\right)$ is analytic and

$$
\left.\left|I_{c}\left(\sigma^{\prime}\right)\right| \leqq C \exp \left(-\frac{1}{8} L^{-(d-1 / 3)} \beta^{2 \alpha}\right)\right) .
$$

Proof of Lemma III.1. Using the global upper bound for complex $\sigma^{\prime},\left|\sigma^{\prime}\right|$ $<\left(2 L^{d-2} \beta\right)^{\alpha}$ we have

where

$$
\left|I_{c}\left(\sigma^{\prime}\right)\right| \leqq \int \exp \left\{-\frac{1}{2}\left(u^{2}+t^{2}\right)+e_{1}+e_{2}\right\} \chi_{c} d u d t,
$$

$$
\begin{aligned}
& e_{1} \equiv-4 \lambda L^{d} u^{2}+4 \lambda L^{d} \operatorname{Re}\left(\frac{L^{-1 / 2(d-2)}}{1+8 \lambda L^{d}} \sigma^{\prime}+u\right)^{2}+\frac{L^{2}}{2} \frac{L^{-(d-2)}}{\left(1+8 \lambda L^{d}\right)^{2}}\left(\operatorname{Im} \sigma^{\prime}\right)^{2}, \\
& e_{2} \equiv-L^{d} \lambda^{*}\left(\left|\left(\frac{L^{-1 / 2(d-2)}}{\left(1+8 \lambda L^{d}\right)} \operatorname{Re} \sigma^{\prime}+u+\beta^{1 / 2}\right) \phi+t\right|-\beta^{1 / 2}\right)^{2} .
\end{aligned}
$$

We estimate $e_{1}$ and $e_{2}$. We have, for $\frac{\lambda^{*}}{2} \leqq \lambda \leqq 3 \lambda^{*}, e_{1} \leqq \delta \beta^{2 \alpha}+\frac{u^{2}}{9}$, where $\delta$ depends only on $L$.

Thus if $|u|$ or $|t| \geqq(12 \delta)^{1 / 2} \beta^{\alpha}, e$, the exponent in Eq. (3.1) is bounded above by

$$
e \leqq-\frac{1}{2}\left(u^{2}+t^{2}\right)+e_{1} \leqq-\frac{1}{4}\left(u^{2}+t^{2}\right)-\frac{1}{12}\left(u^{2}+t^{2}\right)+\delta \beta^{2 \alpha} \leqq-\frac{1}{4}\left(u^{2}+t^{2}\right) .
$$

Now suppose $|u|$ and $|t|<(12 \delta)^{1 / 2} \beta^{\alpha}$, then

$$
\frac{-e_{2}}{L^{d} \lambda^{*}}=\left(\frac{L^{-1 / 2(d-2)}}{1+8 \lambda L^{d}} \operatorname{Re} \sigma^{\prime}+u\right)^{2}+\beta^{3 \alpha-1 / 2} .
$$

Thus

$$
\begin{aligned}
e_{1}+e_{2} \leqq 4 \frac{\lambda}{\lambda^{*}}\left(4 \lambda-\lambda^{*}\right) & \left(\operatorname{Re} \sigma^{\prime}\right)+\frac{L^{2}}{2\left(1+8 \lambda L^{d}\right)^{2}}\left(L^{2-d}-8 \lambda\right)\left(\operatorname{Im} \sigma^{\prime}\right)^{2}+O\left(\beta^{3 \alpha-1 / 2}\right) . \\
\frac{20}{L^{d}}\left|\sigma^{\prime}\right|^{2}+O\left(\beta^{3 \alpha-1 / 2}\right) & \leqq 20 L^{-d}\left(2 L^{d-2} \beta\right)^{2 \alpha}+O\left(\beta^{3 \alpha-1 / 2}\right) \\
& \leqq L^{-(d-1 / 3)} \beta^{2 \alpha}\left[20(2)^{2 \alpha} L^{-1 / 3+2 \alpha(d-2)}+O\left(\beta^{\alpha-1 / 2}\right)\right]
\end{aligned}
$$

for $|u|$ and $|t|<(12 \delta)^{1 / 2} \beta^{\alpha}$. On the other hand, if $|u|$ or $|t|>L^{-1 / 2(d-1 / 3)} \beta^{\alpha}$, then $\frac{1}{4}\left(u^{2}+t^{2}\right) \geqq \frac{1}{4} L^{-(d-1 / 3)} \beta^{2 \alpha}$. Now choose $\alpha<\frac{1}{6(d-2)}, \alpha>0$, and with $\alpha$ fixed choose $L$ so large so that $20(2)^{\alpha} L^{-1 / 3+2 \alpha(d-2)}<\frac{1}{8}$. Now take $\beta$ large so the term $O\left(\beta^{\alpha-1 / 2}\right)$ in Eq. (3.2) is $<\frac{1}{8}$. It then follows that $e_{1}+e_{2}<\frac{1}{2} L^{-(d-1 / 3)} \beta^{2 \alpha}$ and $e_{1}+e_{2}$ $<\frac{1}{4}\left(u^{2}+t^{2}\right)$. But then the exponential of Eq. (3.1) is bounded above by $-\frac{1}{4}\left(u^{2}+t^{2}\right)$ also for $|u|,|t|<(12 \delta)^{1 / 2} \beta^{\alpha}$. In conclusion we have, for $\left|\sigma^{\prime}\right|<\left(2 L^{d-2} \beta\right)^{\alpha}$,

$$
\left|I_{c}\left(\sigma^{\prime}\right)\right| \leqq \int \chi_{c} \exp \left\{-\frac{1}{4}\left(u^{2}+t^{2}\right)\right\} d u d t \leqq c \exp \left(-\frac{1}{8} L^{-(d-1 / 3)} \beta^{2 \alpha}\right),
$$

and the bound implies analyticity of $I_{c}\left(\sigma^{\prime}\right)$. 
Concerning $I_{0}\left(\sigma^{\prime}\right)$ we have

Lemma III.2. For $\left|\sigma^{\prime}\right|<\left(2 L^{d-2} \beta\right)^{\alpha}$

$$
\log I_{0}\left(\sigma^{\prime}\right)=\log I_{0}-g_{1}^{\prime}\left(\sigma^{\prime}\right)
$$

where

$$
I_{0}=\left(1+8 \lambda L^{d}\right)^{-1 / 2}\left(1+O\left(\beta^{3 \alpha-1 / 2}\right) \geqq c>0,\right.
$$

$g_{1}^{\prime}\left(\sigma^{\prime}\right)$ is analytic and $\left|g_{1}^{\prime}\left(\sigma^{\prime}\right)\right| \leqq C \beta^{3 \alpha-1 / 2}$.

Proof of Lemma III.2. We write

$I_{0}\left(\sigma^{\prime}\right)=\int \chi \exp \left\{-\frac{1}{2}\left(1+8 \lambda L^{d}\right) u^{2}-\frac{1}{2} t^{2}+4 \lambda L^{d}\left(\frac{L^{-1 / 2(d-2)}}{\left(1+8 \lambda L^{d}\right)} \sigma^{\prime}+u\right)^{2}-L^{d} V(r)\right\} d u d t$

where we define $r \equiv\left(\frac{L^{-1 / 2(d-2)}}{1+8 \lambda L^{d}} \sigma^{\prime}+u+\beta^{1 / 2}\right) \hat{\phi}+t$

By hypothesis $V$ extends analytically from real $\eta \equiv|r|-\beta^{1 / 2}$ to complex $\eta$ in $|\eta|<\beta^{\alpha} . \eta$ is analytic in $\sigma^{\prime}$ for $\left|\sigma^{\prime}\right|<\left(2 L^{d-2} \beta\right)^{\alpha}$ and $|u|,|t| \leqq L^{-1 / 2(d-1 / 3)} \beta^{\alpha}$ and

$$
\eta=\left(\frac{L^{-1 / 2(d-2)}}{1+8 \lambda L^{d}} \sigma^{\prime}+u\right)+\left(\beta^{2 \alpha-1 / 2}\right)
$$

so that $|\eta|<\beta^{\alpha}$ if $\beta$ is large. Thus $V$ is analytic in $\sigma^{\prime}$ in $\left|\sigma^{\prime}\right|<\left(2 L^{d-2} \beta\right)^{\alpha}$. Now $V(r)$ $=4 \lambda \eta^{2}+w(\eta)$ and letting

$$
\widetilde{V}(r) \equiv V(r)-4 \lambda\left(\frac{L^{-1 / 2(d-2)}}{d} \sigma^{\prime}+u\right)^{2}
$$

we have

$$
|\tilde{V}(r)| \leqq \frac{405}{4} L^{2-d} L^{-3 / 2(d-1 / 3)} \beta^{3 \alpha-1 / 2}+|w(\eta)|+O\left(\beta^{4 \alpha-1}\right),
$$

with $|w(\eta)| \leqq 1100 k L^{-d-1 / 2}\left(2 L^{d-2} \beta\right)^{3 \alpha-1 / 2}$.

Now write $I_{0}\left(\sigma^{\prime}\right)=I_{0} \int e^{-L^{d} \hat{V}(r)} d v$, where

$$
d v=\chi \exp \left(-\frac{1}{2}\left(1+8 \lambda L^{d}\right) u^{2}-\frac{1}{2} t^{2}\right) d u d t /\left(\int \chi \exp (\ldots) d u d t \equiv I_{0}\right) .
$$

The bound for $\tilde{V}$ implies that $I_{0}\left(\sigma^{\prime}\right)$ is analytic on $\left|\sigma^{\prime}\right|<\left(2 L^{d-2} \beta\right)^{\alpha}$. Write

$$
\log I_{0}\left(\sigma^{\prime}\right)=\log I_{0}+\log \left[1+\int\left(e^{-L^{d \hat{V}}(r)}-1\right) d v\right] \equiv \log I_{0}-g_{1}^{\prime}\left(\sigma^{\prime}\right) .
$$

We see that $g_{1}^{\prime}\left(\sigma^{\prime}\right)$ is analytic on $\left|\sigma^{\prime}\right|<\left(2 L^{d-2} \beta\right)^{\alpha}$ and $\left|g_{1}^{\prime}\left(\sigma^{\prime}\right)\right|$ $\leqq 4400 L^{-1 / 2} k\left(2 L^{d-2} \beta\right)^{3 \alpha-1 / 2}$. For $I_{0}$ we have $I_{0}=\left(1+8 \lambda L^{d}\right)^{1 / 2}+O\left(e^{-1 / 4(d-1 / 3)} \beta^{2 \alpha}\right)$ $\geqq \frac{1}{\left(1+24 \lambda^{1} L^{d}\right)^{1 / 2}}+O\left(e^{-1 / 4(d-1 / 3) \beta^{2 \alpha}}\right) \geqq c$, where $c$ is a strictly positive $L$ dependent constant.

Combining Lemmas III.1 and III.2 gives

Lemma III.3. For $\left|\sigma^{\prime}\right|<\left(2 L^{d-2} \beta\right)^{\alpha}$

$$
I\left(\sigma^{\prime}\right)=I_{0} e^{-g^{\prime} 2\left(\sigma^{\prime}\right)},
$$

$g_{2}^{\prime}\left(\sigma^{\prime}\right)$ is analytic and $\left|g_{2}^{\prime}\left(\sigma^{\prime}\right)\right| \leqq O\left(\beta^{3 \alpha-1 / 2}\right)$. 
Returning to the expression for $V^{\prime}(\phi)$ we have $V^{\prime}(\phi)=c+4 \lambda_{L} \sigma^{2}+g_{2}^{\prime}\left(\sigma^{\prime}\right)$. Arguing exactly as in the first step proves that $V^{\prime}(\phi)$ has exactly one minimum, call it $\sigma_{0}$, in the region $\left|\sigma^{\prime}\right|<\left(\frac{3}{2} L^{d-2} \beta\right)^{\alpha}$ and $\sigma_{0}=O\left(\beta^{2 \alpha-1 / 2}\right)$. Taylor expanding $V^{\prime}(\phi)$ around $\sigma_{0}$ we find, for

$$
\left|\sigma^{\prime}-\sigma_{0}\right|<\left(\frac{5}{4} L^{d-2} \beta\right)^{\alpha}, V^{\prime}(\phi)=c+4 \lambda^{\prime} \sigma^{\prime 2}+\sum_{n=3}^{\infty} c_{n}\left(\sigma^{\prime}-\sigma_{0}\right)^{n}
$$

where

$$
\lambda^{\prime}=\lambda_{L}+\frac{1 d^{2}}{8_{d \sigma^{\prime}} 2} g_{2}^{\prime}\left(\sigma_{0}\right), \quad c_{n}=\frac{1 d^{n}}{n !_{d \sigma^{\prime}} n} g_{2}^{\prime}\left(\sigma_{0}\right) .
$$

From Cauchy estimates

$$
\left|c_{n}\right| \leqq 5000 L^{-1 / 2} k\left(2 L^{d-2} \beta\right)^{3 \alpha-1 / 2} \frac{1}{\left(\frac{3}{2} L^{d-2} \beta\right)^{n \alpha}}
$$

so that

$$
\lambda^{\prime}=\lambda_{L}+O\left(\beta^{\alpha-1 / 2}\right)
$$

and, if $L$ is large,

$$
\left|w^{\prime}\left(\sigma^{\prime}-\sigma_{0}\right) \equiv \sum_{n=3}^{\infty} c_{n}\left(\sigma^{\prime}-\sigma_{0}\right)^{n}\right| \leqq k\left(2 L^{d-2} \beta\right)^{3 \alpha-1 / 2} .
$$

Writing $\sigma=\sigma^{\prime}-\sigma_{0}$ and defining $V^{\prime}(\phi)$ to be zero at its minimum $\sigma=0$ we have, for $|\sigma|<\left(\frac{5}{4} L^{d-2} \beta\right)^{\alpha}$,

$$
V^{\prime}=4 \lambda^{\prime} \sigma^{2}+\omega^{\prime}(\sigma) \text {. }
$$

Now $\phi=\left(\sigma^{\prime}+L^{1 / 2(d-2)} \beta^{1 / 2}\right) \phi=\left(\sigma+\sigma_{0}+L^{1 / 2(d-2)} \beta^{1 / 2}\right) \phi \leqq\left(\sigma+\beta^{1 / 2}\right) \phi$, where $\beta^{1 / 2}$ $=L^{1 / 2(d-2)} \beta^{1 / 2}+O\left(\beta^{2 \alpha-1 / 2}\right)<\left(\frac{5}{4}\right)^{1 / 2} L^{1 / 2(d-2)} \beta^{1 / 2}$ for large $\beta$. Thus the region $|\sigma|<\beta^{\prime \alpha}$ is contained in $|\sigma|<\left(\frac{5}{4} L^{d-2} \beta\right)^{\alpha}$ so that the representation given above for $V^{\prime}(\phi)$ is valid in this region and also $\left|\omega^{\prime}(\sigma)\right| \leqq k \beta^{\prime 3 \alpha-1 / 2}$.

We now turn to the proof of the global upper bound for $e^{-V^{\prime}(\phi)}$. With the correct normalization we have

$$
\left.e^{-V^{\prime}(\phi)}=e^{R V\left(\beta^{\prime 1 / 2}\right.} \hat{\phi}\right)_{e}-R V(\phi)
$$

Proceeding as in the first step we have $e^{-R V\left(\beta^{\prime 1 / 2} \hat{\phi}\right)}=e^{-4 \lambda_{L} \sigma^{\prime 2}} I\left(\sigma^{\prime}\right)$, where $\sigma^{\prime}=\beta^{\prime 1 / 2}$ $-L^{1 / 2(d-2)} \beta=O\left(\beta^{2 \alpha-1 / 2}\right)$ and as before, $e^{-R V\left(\beta^{\prime 1 / 2} \hat{\phi}\right)} \geqq C$. Regarding $e^{-R V(\phi)}$ we have, using the global upper bound for $e^{-V}$,

$$
e^{-R V(\phi)} \leqq \int \exp \left[-\lambda^{L} L^{d}\left(|\xi|-\beta^{1 / 2}\right)^{2}-\frac{1}{2}\left(\xi-L^{-1 / 2(d-2)} \phi\right)^{2}\right] d \xi,
$$

and from this point on the proof follows that of the first step. Also the extension to complex $\phi$ is carried out as in the first step.

We have thus completed the proof that if $V$ satisfies properties a)-d), if $L \geqq L_{0}$, if $\beta>\beta_{0}(L)$ and $\frac{1}{2} \lambda^{*} \leqq \lambda \leqq 3 \lambda^{*}$ then $V^{\prime}$ also satisfies a)-d) with $\beta^{1 / 2}=L^{1 / 2(d-2)} \beta^{1 / 2}$ $+O\left(\beta^{2 \alpha-1 / 2}\right), \lambda^{\prime}=\frac{L^{2} \lambda}{1+8 \lambda L^{d}}+O\left(\beta^{\alpha-1 / 2}\right)$. It is easy to verify that $\frac{\lambda^{*}}{2} \leqq \lambda^{\prime} \leqq 3 \lambda^{*}$ if $\beta_{0}$ is large and that $\beta^{\prime 1 / 2}>\frac{1}{2} L^{1 / 2(d-2)}>\beta^{1 / 2}$. Hence the procedure can be repeated.

To complete the proof of Theorem 1 we have to show the existence of the limits $\lim \lambda_{n}, \lim _{n \rightarrow \infty} L^{-n(d-2)} \beta_{n}$ and obtain the representation and bounds for $d_{n}$. We have 
Lemma III.4. The following limits exist:
a) $\lim _{n \rightarrow \infty} \lambda_{n}=\lambda^{*}$,
b) $\lim _{n \rightarrow \infty} L^{-n(d-2)} \beta_{n}$.

Proof of Lemma III.4.a). We have

$$
\lambda_{n+1}=\frac{\lambda_{n} L^{2}}{1+8 \lambda_{n} L^{d}}+O\left(\beta_{n}^{\alpha-1 / 2}\right)
$$

with the $O(\cdot)$ term uniform in $n$.

Thus

$$
\lambda_{n+1}^{-1}-\lambda^{*-1}=\frac{1}{L^{2}}\left(\lambda_{n}^{-1}-\lambda^{*-1}\right)+O\left(\beta_{n}^{\alpha-1 / 2}\right),
$$

which upon iteration gives

$$
\lambda_{n+1}^{-1}-\lambda^{*-1}=L^{-2(n+1)}\left(\lambda_{0}^{-1}-\lambda^{*-1}\right)+\sum_{j=0}^{n} L^{-2(n-j)} O\left(\beta_{j}^{\alpha-1 / 2}\right) .
$$

We have $\beta_{j} \geqq \frac{1}{4} L^{d-2} \beta_{j-1}$ so that $\beta_{j}^{\alpha-1 / 2} \leqq \beta_{0}^{\alpha-1 / 2}\left(\frac{1}{4} L^{d-2}\right)^{(\alpha-1 / 2) j}$ and

$$
S_{n} \equiv \frac{1}{L^{2 n}} \sum_{j=0}^{n} L^{2 j} \beta_{j}^{(\alpha-1 / 2)} \leqq \frac{\beta_{0}^{\alpha-1 / 2}}{L^{2 n}} \sum_{j=0}^{n}\left[L^{2}\left(\frac{1}{4} L^{d-2}\right)^{\alpha-1 / 2}\right]^{j} .
$$

Now $\alpha<\frac{1}{6(d-2)}$ implies $\left(\frac{1}{4} L^{d-2}\right)^{\alpha-1 / 2} \leqq 2 L^{-1 / 3}$ so that $S_{n} \underset{n \rightarrow \infty}{\longrightarrow} O$.

Proof of Lemma III.4.b). We have $\beta_{n+1}^{1 / 2}=L^{1 / 2(d-2)} \beta_{n}^{1 / 2}+O\left(\beta_{n}^{2 \alpha-1 / 2}\right)$ with the $O(\cdot)$ term uniform in $n$. Iterating gives

$$
\begin{aligned}
& \left|L^{-1 / 2(n+k)(d-2)} \beta_{n+k}^{1 / 2}-L^{-1 / 2 n(d-2)} \beta_{n}^{1 / 2}\right| \\
& \quad=\left|L^{-1 / 2(n+k)(d-2)} O\left(\beta_{n+k-1}^{2 \alpha-1 / 2}\right)+\ldots+L^{-1 / 2(n+1)(d-2)} O\left(\beta_{n}^{2 \alpha-1 / 2}\right)\right| \\
& \quad \leqq \frac{c(L)}{\beta_{n}^{1 / 2-\alpha}} \sum_{j=1}^{k} L^{-1 / 2(n+j)(d-2)} \stackrel{n \rightarrow \infty}{\longrightarrow} 0 . \quad \square
\end{aligned}
$$

To obtain the representation and bounds for $d_{n}$ let $e^{-V^{(n+1)}}=c_{n} e^{-R V^{(n)}}$. By induction we easily find that

$$
e^{-V^{(n)}(\phi)}=\exp \left[\sum_{j=0}^{n-1} L^{(n-1-j) d} \log c_{j}\right] e^{-R^{n} V^{(0)}}(\phi)
$$

So that $d_{n}=\sum_{j=0}^{n-1} L^{(n-1-j) d} \log c_{j}$.

Now $c_{j}^{-1}=e^{-R V^{(j)}}\left(\beta_{j+1}^{1 / 2} \phi\right)$ and the integral has been estimated in the Proof of Lemmas II. 2 and III.2 leading to the value

$$
c_{j}=\left(1+8 \lambda_{j} L^{d}\right)^{1 / 2}\left(1+O\left(\beta_{j}^{3 \alpha-1 / 2}\right)\right) .
$$

Thus the proof of Theorem 1 is complete. 


\section{Free energy for $h=0$. Proof of Theorem 2.}

We will show below that

$$
\lim _{N \rightarrow \infty} L^{-N d} \operatorname{In} I_{N}=0
$$

where

$$
I_{n}=\int e^{-V^{(N)}(\phi)} d \mu(\phi) \text { so that } F=\lim _{N \rightarrow \infty} \beta^{-1} L^{-N d} d_{N} .
$$

Using Theorem $1 \mathrm{f}$ gives the representation for $F$.

We now turn to the bounds on $I$ which imply Eq. (4.1). We have

\section{Lemma 4.1.}

$$
c e^{-\left[4 \lambda_{N}+\left(1-L^{2-d}\right)\right] \beta} N \leqq I_{N} \leqq 1 .
$$

Proof of Lemma 4.1. The upper bound follows from the global upper bound for $e^{V^{(N)}(\phi)}$. For the lower bound letting $\chi_{0}$ be the characteristic function of the set $\left\{\phi \in R^{v}:|| \phi\left|-\beta_{N}^{1 / 2}\right|<\beta_{N}^{\alpha}\right\}$ and using Theorem I gives

$$
\begin{aligned}
I_{N} & \geqq c \int \chi_{0} e^{-4 \lambda_{N}\left(|\phi|-\beta_{N}^{1 / 2}\right)^{2}-w_{N}\left(|\phi|-\beta_{N}^{1 / 2}\right)-1 / 2\left(1-L^{2-d}\right)|\phi|^{2}} d \phi \\
& \geqq C \beta_{N}^{(1 / 2(v-1)+\alpha)} \int \exp \left[-4 \lambda_{N} \sigma^{2}-1 / 2\left(1-L^{2-d}\right)\left(\sigma+\beta_{N}^{1 / 2}\right)^{2}\right] d v(\sigma),
\end{aligned}
$$

where $d v(\sigma)=\chi_{0}(\sigma) d \sigma / \int \chi_{0}(\sigma) d \sigma$. Thus by Jensen's inequality

$$
I_{N} \geqq C \exp \left[-4 \lambda_{N}\left\langle\sigma^{2}\right\rangle-\frac{1}{2}\left(1-L^{2-d}\right)\left(\langle\sigma\rangle^{2}+\beta_{N}\right)\right],
$$

where $\langle\cdot\rangle$ is with respect to $d v$. Using $\left\langle\sigma^{2}\right\rangle \leqq \beta_{N}^{2 \alpha}<\beta_{N}$ the lowerbound follows.

\section{Proof of Spontaneous Magnetization and Effective Action Limit}

We prove Theorem 3; first the result for $m$, then the equality $F_{+}=F$. Finally we give the proof of Theorem 4.

Using the shift formula for $R^{N}\left(V-\beta^{1 / 2} h_{N} I_{1}\right)$ we obtain the representation

$$
m_{N}=-L^{2 N+d}\left(\frac{1-L^{-2 N}}{L^{2}-1}\right) h_{N}+\frac{L^{-N d}}{\beta} Z_{N}^{\prime-1} \frac{\partial Z_{N}^{\prime}}{\partial h},
$$

where

$$
\begin{aligned}
Z_{N}^{\prime}= & \int \exp \left[-V^{(N)} \phi\left(+L^{d} L^{1 / 2 N(d+2)}\left(\frac{1-L^{2 N}}{L^{2-1}}\right) \beta^{1 / 2} h_{N} e_{1}\right)\right. \\
& \left.+L^{1 / 2 N(d+2)} \beta^{1 / 2} h_{N} \phi_{1}-\frac{1}{2}\left(1-L^{2-d}\right) \phi^{2}\right] d \phi .
\end{aligned}
$$

Making a translation in the above integral gives

$$
\begin{aligned}
m_{N}= & -L^{2 N+d}\left(\frac{1-L^{-2 N}}{L^{2}-1}\right)\left[\frac{L^{d-1}}{L^{2}-1}-\left(\frac{L^{d}-L^{2}}{L^{2}-1}\right) L^{2 N}\right] h_{N} \\
& +\left[\frac{L^{d-1}}{L^{2}-1}-\left(\frac{L^{d}-L^{2}}{L^{2}-1}\right) L^{-2 N}\right] \beta^{-1 / 2} L^{-1 / 2 N(d-2)}\left\langle\phi_{1}\right\rangle_{U_{N}}
\end{aligned}
$$


where

$$
\langle\cdot\rangle_{U_{N}} \equiv \int \cdot e^{-U_{N}} d \phi / \int e^{-U_{N}} d \phi
$$

with

$$
\begin{aligned}
U_{N} & =V^{(N)}(\phi)-\left[\frac{L^{d-1}}{L^{2}-1}-\left(\frac{L^{d}-L^{2}}{L^{2}-1}\right) L^{-2 N}\right] L^{1 / 2 N(d+2)} \beta^{1 / 2} h_{N} \phi_{1} \\
& +\frac{1}{2}\left(1-L^{2-d}\right) \phi^{2} .
\end{aligned}
$$

We now show that the result for $m$ follows from

\section{Lemma 5.1.}

$$
\left\langle\phi_{1}\right\rangle_{U_{N}}=\beta_{N}^{1 / 2}\left(1+O\left(\beta_{N}^{3 \alpha-1 / 2}\right)\right) .
$$

Using Eq. (5.3) in Eq. (5.1) gives

$$
\begin{aligned}
m_{N}= & \beta^{-1 / 2} L^{-1 / 2 N(d-2)} \beta_{N}^{1 / 2}\left\{\left[\frac{L^{d}-1}{L^{2}-1}-\left(\frac{L^{d}-L^{2}}{L^{2}-1}\right) L^{-2 N}\right]\left(1+O\left(\beta_{N}^{3 \alpha-1 / 2}\right)\right)\right. \\
& \left.-\left(L^{d}-L^{2}\right)\left(\frac{1-L^{-2 N}}{L^{2}-1}\right)\right\}
\end{aligned}
$$

so that

$$
\begin{aligned}
m & =\lim _{N \rightarrow \infty} m_{N}=\beta^{-1 / 2} \lim _{N \rightarrow \infty} L^{-1 / 2 N(d-2)} \beta_{N}^{1 / 2}\left\{\frac{L^{d}-1}{L^{2}-1}-\frac{L^{d}-L^{2}}{L^{2}-1}\right\} \\
& =\beta^{-1 / 2} \lim _{N \rightarrow \infty} L^{-N / 2(d-2)} \beta_{N}^{1 / 2} .
\end{aligned}
$$

Proof of Lemma 5.1. Using the formula for $h_{N}$ we have

$$
\left\langle\phi_{1}\right\rangle_{U_{N}}=\int \phi_{1} e^{-\tilde{U}_{N}(\phi)} d \phi / \int e^{-\tilde{U}_{N}(\phi)} d \phi \equiv N / D,
$$

where

$$
\tilde{U}_{N}=V^{(N)}+\frac{1}{2}\left(1-L^{2-d}\right) \phi_{\perp}^{2}+\frac{1}{2}\left(1-L^{2-d}\right)\left(\phi_{1}-\beta_{N}^{1 / 2}\right)^{2} .
$$

To estimate $N$ write $N=N_{1}+N_{2}$, where $N_{1}=\int \chi \phi_{1} e^{-\tilde{U}_{N}} d \phi$ and

$$
\chi(\phi)= \begin{cases}1, & \text { if }\left|\phi_{1}-\beta_{N}^{1 / 2}\right|<1 / 2 \beta_{N}^{\alpha} \text { and }\left|\phi_{\perp}\right|<\frac{1}{2} \beta_{N}^{\alpha}, \phi_{\perp} \equiv \phi_{1}-\phi_{1} \hat{e}_{1} \\ 0, & \text { otherwise }\end{cases}
$$

In $N_{1}$ we can use the small field representation $V^{(N)}$ from Theorem 1. Let $F_{N}=4 \lambda_{N}\left(|\phi|-\beta_{N}^{1 / 2}\right)^{2}+\frac{1}{2}\left(1-L^{2-d}\right) \phi_{\perp}^{2}+\frac{1}{2}\left(1-L^{2-d}\right)\left(\phi_{1}-\beta_{N}^{1 / 2}\right)^{2}$, then $F_{N}$ has an absolute minimum at $\phi_{1}=\beta_{N}^{1 / 2}, \phi_{\perp}=0$. Taylor expanding $F_{N}$ and estimating the remainder gives

$$
N_{1}=\int \phi_{1} \chi \exp \left\{-\frac{1}{2}\left[8 \lambda_{N}+\left(1-L^{2-d}\right)\right]\left(\phi_{1}-\beta_{N}^{1 / 2}\right)^{2}-\frac{1}{2}\left(1-L^{2-d}\right) \phi_{\perp}^{2}+G_{N}\right\} d \phi
$$

where $G_{N}=O\left(\beta_{N}^{3 \alpha-1 / 2}\right)$ uniformly in $\chi(\phi)=1$. In Eq. (5.3) write $e^{G_{N}}=e^{G_{N}}+\left(e^{G_{N}}-1\right)$ with the corresponding decomposition $N_{1}=N_{1}^{\prime}+N_{1}^{\prime \prime}$. Thus $N_{1}^{\prime \prime}=O\left(\beta_{N}^{3 \alpha-1 / 2}\right) N_{1}^{\prime}$ and $N_{1}=\left(1+O\left(\beta_{N}^{3 \alpha-1 / 2}\right)\right) N_{1}^{\prime}$. In $N_{1}^{\prime}$ write $\chi=1-\chi_{c}$ and $\phi_{1}=\phi_{1}-\beta_{N}^{1 / 2}+\beta_{N}^{1 / 2}$ which gives

$$
\begin{aligned}
N_{1}= & \beta_{N}^{1 / 2} \int \exp \left[-\frac{1}{2}\left(8 \lambda_{N}+\left(1-L^{2-d}\right)\right)\left(\phi_{1}-\beta_{N}^{1 / 2}\right)^{2}\right. \\
& \left.-\frac{1}{2}\left(1-L^{2-d}\right) \phi_{\perp}^{2}\right] d \phi \cdot\left(1+O\left(\beta_{N}^{3 \alpha-1 / 2}\right)\right)
\end{aligned}
$$


We now estimate $N_{2}$. Using the global upper bound of Theorem 1 we have

$$
\begin{aligned}
\left|N_{2}\right| & \leqq \exp \left[-\frac{1}{16}\left(1-L^{2-d}\right) \beta_{N}^{2 \alpha}\right] \int\left|\phi_{1}\right| \exp \left[-\frac{1}{4}\left(1-L^{2-d}\right)\left(\phi-\beta_{N} e_{1}\right)^{2}\right] d \phi \\
& \leqq C\left(1+\beta_{N}^{1 / 2}\right) e^{-1 / 16\left(1-L^{2-d}\right) \beta_{N}^{2 \alpha}} .
\end{aligned}
$$

Thus

$$
\begin{gathered}
N=N_{1}+N_{2}=\beta_{N}^{1 / 2} \int \exp \left[-\frac{1}{2}\left(8 \lambda_{N}+\left(1-L^{2-d}\right)\right)\left(\phi_{1}-\beta_{N}^{1 / 2}\right)^{2}\right. \\
\left.-\frac{1}{2}\left(1-L^{2-d}\right) \phi_{\perp}^{2}\right] d \phi \cdot\left(1+O\left(\beta_{N}^{3 \alpha-1 / 2}\right)\right) .
\end{gathered}
$$

A similar analysis of $D$ leads to

$$
\begin{aligned}
D= & \int \exp \left[-\frac{1}{2}\left(8 \lambda_{N}+\left(1-L^{2-d}\right)\right)\left(\phi_{1}-\beta_{N}^{1 / 2}\right)^{2}\right. \\
& \left.-\frac{1}{2}\left(1-L^{2-d}\right) \phi_{\perp}^{2}\right] d \phi \cdot\left(1+O\left(\beta_{N}^{3 / 2-1 / 2}\right)\right)
\end{aligned}
$$

and the proof of the lemma is complete.

We now prove the equality $F_{+}=F \cdot F_{N}\left(h_{N}\right)$ is given by

$$
\begin{aligned}
F_{N}\left(h_{N}\right)= & -\frac{1}{2} L^{d+2 N}\left(\frac{1-L^{-2 N}}{L^{2}-1}\right) h_{N}^{2}+\frac{d_{N}}{\beta L^{N d}}-\frac{C}{\beta L^{N d}} \\
& -\frac{1}{\beta L^{N d}} \log \int \exp \left[-V^{(N)}\left(\phi+L^{d} L^{1 / 2 N(d+2)}\left(\frac{1-L^{-2 N}}{L^{2}-1}\right) \beta^{1 / 2} h_{N} \hat{e}_{1}\right)\right. \\
& \left.+L^{1 / 2 N(d+2)} \beta^{1 / 2} h_{N} \phi^{1}-\frac{1}{2}\left(1-L^{2-d}\right) \phi^{2}\right] d \phi,
\end{aligned}
$$

which after translation in the integral becomes

$$
\begin{aligned}
F_{N}\left(h_{N}\right)= & \frac{1}{2} L^{d+2 N}\left(\frac{1-L^{-2 N}}{L^{2}-1}\right)\left[\frac{L^{d}-1}{L^{2}-1}\left(\frac{L^{d}-L^{2}}{L^{2}-1}\right) L^{-2 N}\right] h_{N}^{2} \\
& +\frac{d_{N}}{\beta L^{N d}}-\frac{C}{\beta L^{N d}}-\frac{1}{\beta L^{N d}} \log \int e^{-U_{N}} d \phi,
\end{aligned}
$$

where $U_{N}$ is given by Eq. (5.2).

As in the proof of Lemma 5.1,

$$
\int e^{-U_{N}} d \phi=\exp \left[\frac{1}{2}\left(1-L^{2-d}\right) \beta_{N}\right] \int e^{-\tilde{U}_{N}} d \phi
$$

and $\int e^{-\tilde{U}_{N}} d \phi \equiv D=O(1)$. Noting that $h_{N} \sim L^{-2 N}$ for $N$ large we see that

$$
\lim _{N \rightarrow \infty} F_{N}\left(h_{N}\right)=\frac{1}{\beta} \lim _{N \rightarrow \infty} \frac{d_{N}}{L^{N d}}
$$

and the proof of $F_{+}=F$ and Theorem 3 is complete.

We now turn to the proof of Theorem 4. By Eq. (1.8)

$$
V_{-\beta^{1 / 2} m e_{1}}^{(n)}(\phi)=V^{(n)}\left(\phi+L^{1 / 2(d-2)} \beta^{1 / 2} m e_{1}\right) \text {. }
$$

We first establish a lemma which permits us to use the small field representation Theorem 1 for $V_{-\beta^{1 / 2} m e_{1}}^{(n)}$. We have 
Lemma 5.2. Let $|\phi|<\beta_{n}^{\alpha / 2}$. Then for large $n$

a) $\left|\phi+L^{n / 2(d-2)} \beta^{1 / 2} m \hat{e}_{1}\right|-\beta_{n}^{1 / 2}=\phi_{1}+\left(L^{n / 2(d-2)} \beta^{1 / 2} m-\beta_{n}^{1 / 2}\right)$

$$
+O\left(L^{(2 \alpha-1 / 2) n(d-2)}\right)
$$

b) ||$\phi+L^{n / 2(d-2)} \beta^{1 / 2} m \hat{e}_{1}\left|-\beta_{n}^{1 / 2}\right|<\beta_{n}^{\alpha}$.

Proof of Lemma 5.2.

a) Writing $\phi=\phi_{1} \hat{e}_{1}+t$ with $t \cdot \hat{e}_{1}=0$ we have

$$
\left|\phi+L^{n / 2(d-2)} \beta^{1 / 2} m \hat{e}_{1}\right|=L^{n / 2(d-2)} \beta^{1 / 2} m\left[1+\frac{2}{L^{n / 2(d-2)} \beta^{1 / 2} m} \phi_{1}+\frac{\phi^{2}}{L^{n(d-2)} \beta m^{2}}\right]^{1 / 2} .
$$

Since $\lim _{n \rightarrow \infty} L^{-n / 2(d-2)} \beta_{n}^{1 / 2}$ exists then $|\phi|<L^{n \alpha(d-2)}$ and for large $n$

$$
\left|\frac{2 \phi}{L^{n / 2(d-2)} \beta^{1 / 2} m}+\frac{\phi^{2}}{L^{n(d-2)} \beta m^{2}}\right|<1 .
$$

Thus

$$
\left|\phi+L^{n / 2(d-2)} \beta^{1 / 2} m \hat{e}_{1}\right|=L^{n / 2(d-2)} \beta^{1 / 2} m+\phi_{1}+O\left(L^{(2 \alpha-1 / 2) n(d-2)}\right) .
$$

b) From the proof of Theorem 1,

$$
\begin{aligned}
& \left|L^{-1 / 2(n+k)(d-2)} \beta_{n+k}^{1 / 2}-L^{-n / 2(d-2)} \beta_{n}^{1 / 2}\right| \\
& \leqq \frac{O(1)}{\beta_{n}^{(1 / 2-\alpha)}} L^{-n / 2(d-2)} \sum_{j=1}^{\infty} L^{-j / 2(d-2)}
\end{aligned}
$$

so that

$$
\left|L^{n / 2(d-2)} \beta^{1 / 2} m-\beta_{n}^{1 / 2}\right| \leqq \frac{O(1)}{\beta_{n}^{1 / 2-\alpha}} \sum_{j=1}^{\infty} L^{-j / 2(d-2)},
$$

showing that $\lim _{n \rightarrow \infty}\left(L^{n / 2(d-2)} \beta^{1 / 2} m-\beta_{n}^{1 / 2}\right)=0$. From this and a) we see that for $|\phi|<\beta_{n}^{\alpha} / 2$ and for large $n$ that $\left|\phi+L^{n / 2(d-2)} \beta^{1 / 2} m \hat{e}_{1}\right|-\beta_{n}^{1 / 2} \mid<\beta_{n}^{\alpha}$.

Now suppose $\phi$ belongs to a compact set $B$ contained in a ball of radius $R$. Let $n$ be so large that $\beta_{n}^{\alpha}>2 R$, then by Lemma $5.2 \mathrm{~b}$ ) we can use the small field representation of Theorem 1, i.e.

$$
\begin{aligned}
V_{-\beta^{1 / 2} \hat{e}_{1}}^{(n)}(\phi)= & 4 \lambda_{n}\left(\left|\phi+L^{n / 2(d-2)} \beta^{1 / 2} m \hat{e}_{1}\right|-\beta_{n}^{1 / 2}\right)^{2} \\
& +w_{n}\left(\left|\phi+L^{n / 2(d-2)} \beta^{1 / 2} m e_{1}\right|-\beta_{n}^{1 / 2}\right) .
\end{aligned}
$$

From Lemma 5.2a) for large $n$,

$$
|| \phi+L^{n / 2(d-2)} \beta^{1 / 2} m \hat{e}_{1}\left|-\beta_{n}^{1 / 2}\right| \leqq R+1
$$

and

$$
|| \phi+L^{n / 2(d-2)} \beta^{1 / 2} m e_{1}\left|-\beta_{n}^{1 / 2}\right|^{2}-\phi_{1}^{2} \mid \leqq R r_{n}
$$

with $r_{n} 0$ as $n \rightarrow \infty$ for all $\phi \in B$. Thus

$$
\begin{aligned}
& \left|V_{-\beta^{1 / 2} \hat{e}_{1}}^{(n)}(\phi)-4 \lambda^{*} \phi_{1}^{2}\right| \leqq 4\left|\lambda_{n}-\lambda^{*}\right|\left(\left|\phi+L^{n / 2(d-2)} \beta^{1 / 2} m \hat{e}_{1}\right|-\beta_{n}^{1 / 2}\right)^{2} \\
& \quad+4 \lambda^{*}\left|\left[\left(\mid \phi+L^{n / 2(d-2)} \beta^{1 / 2} m \hat{e}_{1}-\beta_{n}^{1 / 2}\right)^{2}-\phi_{1}^{2}\right]\right|+\left|w_{n}\right| \\
& \leqq 4(R+1)^{2}\left|\lambda_{n}-\lambda^{*}\right|+4 \lambda^{*} R r_{n}+k \beta_{n}^{3 \alpha-1 / 2} \stackrel{n \rightarrow \infty}{\longrightarrow} 0
\end{aligned}
$$

uniformly for $\phi \in B$ and the proof of Theorem 4 is complete. 


\section{Concluding Remarks}

It would be interesting to know the lower bound on the sequence $\left\{h_{N}\right\}$ which will still result in a non-zero spontaneous magnetization.

We considered in this paper a specific sequence of magnetic fields $\left\{h_{N}\right\}$ which produce a pure state in the thermodynamic limit $(N \rightarrow \infty)$. It would be interesting to characterize sequences $\left\{h_{N}\right\}$ which produce mixed states, with the spontaneous magnetization ranging between zero and its maximum (pure state) value. We have obtained in [14] the behavior of the pure state correlation functions which gives the complete Goldstone picture.

\section{References}

1. Domb, C., Green, M.S.: Phase transitions and critical phenomena. Vol. 3. New York: Academic Press 1974

2. Parisi, G.: Statistical field theory. New York: Addison-Wesley 1987

3. Fröhlich, J., Spencer, T.: Commun. Math. Phys. 81, 527 (1981)

4. Fröhlich, J., Spencer, T.: Commun. Math. Phys. 83, 411 (1982)

5. Bricmont, J., Fontaine, J.-R., Lebowitz, J.L., Lieb, E.H., Spencer, T.: Commun. Math. Phys. 78, 545 (1981)

6. Fröhlich, J., Simon, B., Spencer, T.: Commun. Math. Phys. 50, 79 (1976)

7. Balaban, T.: Renormalization group approach to lattice field theories I. Commun. Math. Phys. 109, 249-301 (1987)

8. Balaban, T.: Large field renormalization II. Commun. Math. Phys. 122, 355-392 (1989)

9. Gawedzki, K., Kupiainen, A.: Continuum limit of the hierarchical $O(N)$ non-linear $\sigma$-model. Commun. Math. Phys. 106, 533-550 (1986)

10. Gawedzki, K., Kupiainen, A.: Asymptotic freedom beyond perturbation theory. In: Les Houches Session XLII, 1984. Phénomènes critiques, Systémes aléatoires, Théories de Jauge Elsevier Science Publishers, B.V. 1986, Osterwalder, K., Stora, R. (eds.)

11. Collet, P., Eckmann, J.P.: A renormalization group analysis of the hierarchical model in statistical mechanics. Lecture Notes in Physics. Vol. 74. Berlin, Heidelberg, New York: Springer 1978

12. Bleher, P.M., Major, P.: The large-scale limit of Dyson's hierarchical vector-valued model at low temperatures. Preprint Keldysh Institute of Applied Mathematics. Moscow A-47, 1989

13. Bleher, P.M., Major, P.: The large-scale limit of Dyson's hierarchical vector-valued model at low temperatures. The non-Gaussian case. Ann. Inst. Henri Poincaré, Phys. Théor. 49, Vol. 1 (1988)

14. Schor, R., O'Carroll, M.: Correlation functions and the Goldstone picture for the hierarachical classical Vector model at low temperatures in three or more dimensions. June 1990 (to appear in J. Stat. Phys.)

Communicated by M. Aizenman 
